\title{
Multilayer nonwoven fabrics for filtration of micron and submicron particles
}

\begin{abstract}
Multi layer non woven fabrics were developed by thermal bonding with polyester, cotton and viscose and with the binding fibre as polypropylene. Different proportion of the fibres are used in three different layers and polyester, cotton and viscose were used as top, middle and bottom layers respectively. The thickness and areal density of the fabric was varied depends on the proportion of each layer due to the density variation and shape factor of the fibre. The theoretical solid volume fraction matches the filtration efficiency of the developed multi layer nonwoven fabrics. The highest proportion of viscose fibre as bottom layer leads to reduction of air permeability and better filtration efficiency for the particles of 0.3 to $1 \mu$. Equal proportions of polyester, cotton and viscose fibres give higher air permeability and also filtration efficiency was higher for particles $2-3 \mu$ particles.
\end{abstract}

Keywords: multi layer nonwoven fabric, air filtration, thermal bonding, solid volume fraction
Volume 5 Issue 2 - 2019

\author{
Gobi N, Evangelin S, Kasthuri R, Nivetha D
}

Department of Textile technology, Anna University, India

Correspondence: Gobi Nallathambi, Department of Textile Technology, Anna University, Chennai,Tamil Nadu, India,Tel +9 9884845999, Email gobsnn@gmail.com

Received: August 27, 2018| Published: March 06, 2019

\section{Introduction}

Filtration is the process of separation of dispersed particles from a dispersing fluid such as air or liquid with the help of a porous medium.

Nonwoven fabrics are widely used for filtration of particles due to higher air permeability, better filtration performance and no slippage of yarn than woven filter media. ${ }^{2-5}$ Due to the random fibrous web of the nonwoven fabric, effective filtration was achieved. The filtration performance of the nonwoven fabric was influenced by thickness of the fabric, areal density and pore size. A majority of nonwoven fabrics are spun bonded and in general, the mechanically bonded nonwoven fabrics are thicker, with larger pore opening than thermal bonded nonwoven fabric. Needle punched nonwoven fabrics are used to filter coarse particles such as separations of coal, dust and chemical particles. Other nonwoven based on melt blown, spun bond and needle felting techniques are used in disposable cartridge, membrane and for food filtration. The mean flow pore diameter was achieved as for non-thermal bonded fabrics as 1-500 microns and it was reduced as $10-15$ micron by thermal calendaring. Thermal bonded staple fibre nonwoven fabrics were developed for depth filtration and its performance was better than meltblown nonwoven fabric. ${ }^{6}$

The nonwoven fabrics developed from continuous filaments followed an ordered formation of pores and in case of staple fibres, random arrangement of fibres and higher pore tightness can be achieved. ${ }^{7}$ A random arrangement of fibres in nonwoven provides more favourable conditions for the trapping and precipitation of medium to fine particles. Fibre cross section also influences the air permeability and filtration efficiency of the nonwoven fabric, ${ }^{8}$ the irregular and serrated cross section of fibres can give better filtration performance. The air permeability significantly decreases for thermally bonded nonwoven fabrics when the fabric areal density increases ${ }^{9}$ and it also increases with products from thermal bonded polyester than with those from viscose with low melting bonding fibres. ${ }^{10}$
Thermal bonding may be the area and point bonding, and the area bonding is suitable for staple fibre non woven fabric. In case of thermal bonding, if the mass per unit area increases, the number of melted fibres increases, thus a finer pore size distribution is created. For nonwoven fabrics, increasing the solid volume fraction will generally increase both filtration efficiency and pressure drop. ${ }^{11}$

The use of fibre blends can also improve filter properties by increasing the filtration depth and creating three dimensional structure. ${ }^{12,13}$ Single layer needle punched nonwoven materials in air filtration application include non uniformity of web and distribution size of pores which result in not all the dust particles being intercepted. ${ }^{14}$ A multi layer homogeneous nonwoven fabric can be produced cost effectively with improved filtration performance, cotton and other natural fibres are suitable fibre for making multi layer nonwoven fabric filter. The structural anisotropy of different fibres leads to improve various physical and filtration property of the multilayer nonwoven filter fabrics. Multi layer nonwoven fabrics were used for gas turbine intake filter and it results better performance than the single layer filters. ${ }^{15}$ Mixed fibre filters were also developed for filtering mono and polydisperse aerosol particles and its performance was compared. ${ }^{16}$ For thermal bonding by calendaring, polypropylene is a suitable fibre for bonding of staple fibres. ${ }^{17,18}$ In this study, multilayer non woven fabrics with polyester, cotton and viscose was developed by thermal bonding with the binding fibre as polypropylene and also the filtration performance of articles from 0.3 to $2 \mu$ were analysed.

\section{Materials and methods}

\section{Materials}

Polyester, cotton, viscose and polypropylene staple fibres are used for developing multi layer nonwoven fabrics (MLNF) and the fibre parameters are given in Table 1. Polypropylene was used as a binder fibre and this was mixed with carrier fibre by carding machine and blended web of each fibre was produced. 
Table I Fibre properties

\begin{tabular}{lll}
\hline Fibre & $\begin{array}{l}\text { Length } \\
\mathbf{m m}\end{array}$ & $\begin{array}{l}\text { Denierl } \\
\text { micronaire }\end{array}$ \\
\hline Cotton & 30 & 3.4 \\
Polyester & 32 & 1.2 \\
Viscose & 38 & 1.5 \\
Polypropylene & 32 & 1.2 \\
\hline
\end{tabular}

\section{Construction of multilayer nonwoven fabric}

The principle of construction of multi layer non woven fabric for filtration is shown in Figure 1. Miniature carding machine was used to produce the web with blended the carrier fibre and binder fibre, $25 \%$ of polypropylene on the weight of carrier fibre was used as binder fibre to produce each layer of polyester, cotton and viscose. The experimental plan for the development of multilayer nonwoven fabric preparation was shown in Table 2. The as prepared multi layers of web was thermal bonded at the temperature of $120^{\circ} \mathrm{C}$ for $30 \mathrm{~s}$ by miniature thermal bonding machine.

The surface morphology of the each fibre contributes in filtration performance of non woven fabric and is shown in Figure 2.

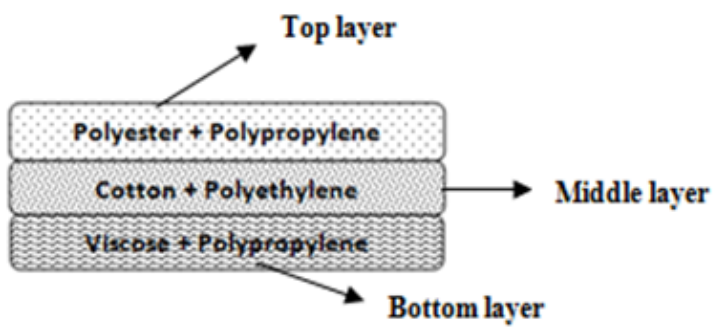

Figure I Principle of the construction of multi layer non woven fabric

Table 2 Physical and filtration properties of multi layer nonwoven fabrics

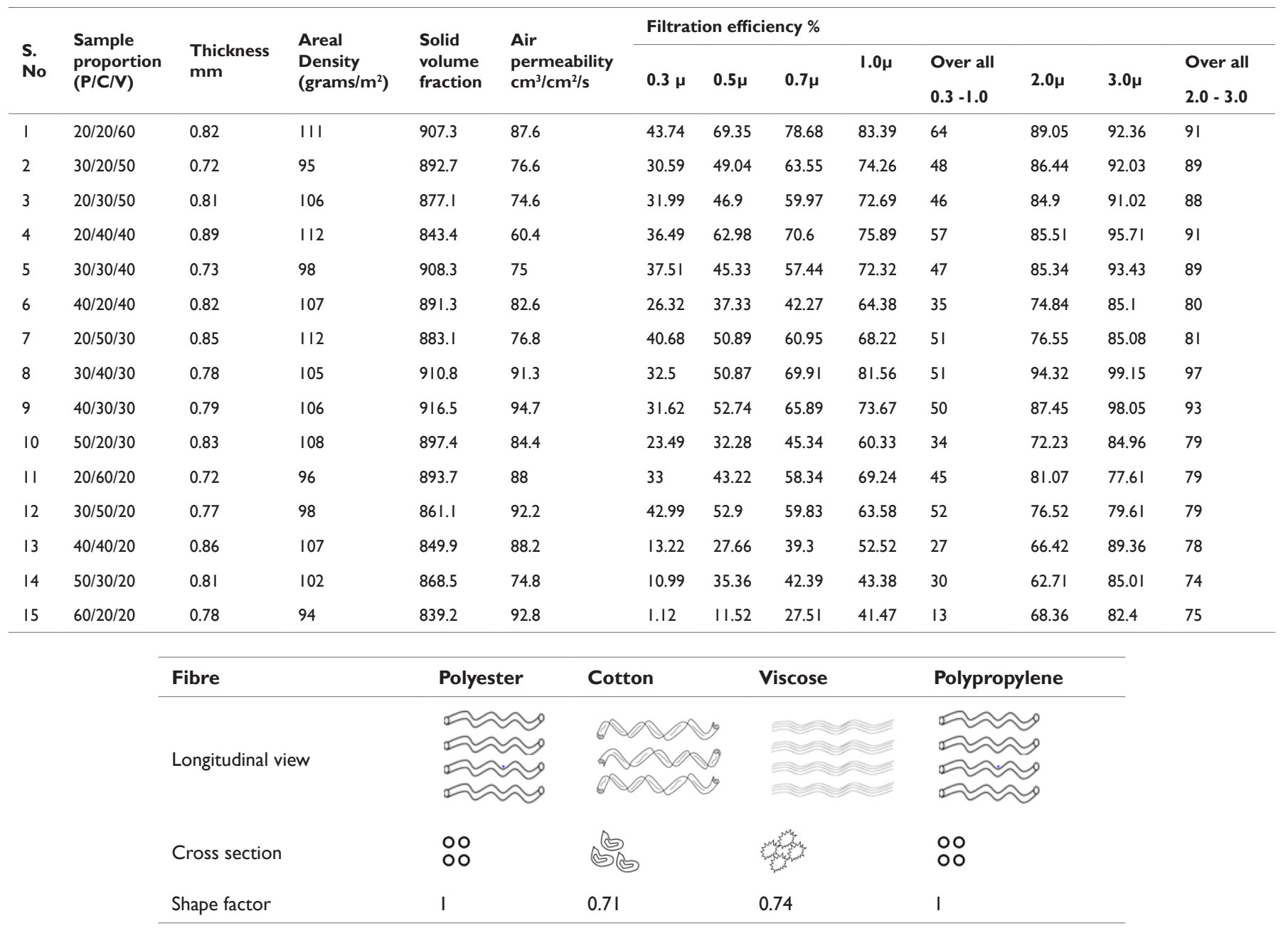

Figure 2 Surface morphology of fibres.

\section{Characterisation of multilayer nonwoven fabric}

The physical properties of the as developed nonwoven fabric were analysed as per the standard test methods. The areal density of the fabric was measured by ASTM D 6242-98 and the air permeability of the fabric was measured by ASTM D 737-04 (2016). The solid volume fraction $(\alpha)$ of the non woven fabric was calculated by equation 1 .

$$
\alpha=10 \times \frac{G}{\rho_{f} \times Z}
$$

Where $\mathrm{G}=$ mass per unit area, $\mathrm{g} / \mathrm{m}^{2}, \rho_{\mathrm{f}=}$ fibre density, $\mathrm{g} / \mathrm{cm}^{3}, \mathrm{Z}=$ thickness of the fabric, $\mathrm{mm}$ 


\section{Analysis of filtration efficiency}

The principle of air filtration efficiency tester as per ASTM F229903 was shown in Figure 3. The dry and clean air was obtained from compressor and fed to the air regulator. $\mathrm{NaCl}$ was used to generate aerosol and the size of the aerosol ranging from 0.3 to 10 micron. The aerosol was passed through the neutraliser (P-120) and diluted before passing through the filtration chamber. Sample of $80 \mathrm{~mm}$ diameter of multilayer nonwoven fabric was fixed between the holders. The velocity of the air flow with aerosol was maintained as $5 \mathrm{~cm} / \mathrm{s}$ and the pressure difference between the up and down stream was continuously monitored. Further increase in face velocity will decrease the filtration efficiency of the nonwoven fabrics. Particle size was measured by laser particle counter (Lasair III, Particle measuring system) from up and down air stream to calculate the filtration efficiency. The air filtration efficiency is calculated by equation 2 .

$$
\text { Air filtration efficiency, } \%=[(A-B) /(A)] \times 100
$$

Where $\mathrm{A}=$ number of particles feed, $\mathrm{B}=$ number of particles received after filtration

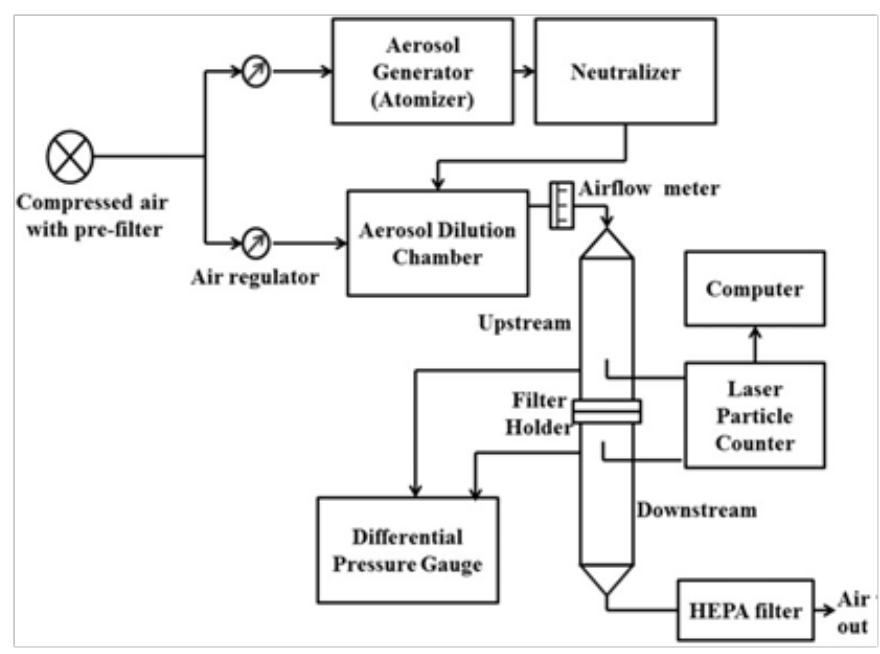

Figure 3 Aerosol filtration efficiency tester.

\section{Results and discussion}

\section{Influence of process variables on areal density and thickness of nonwoven fabrics}

The areal density of the multi layer nonwoven fabric has been ranged between 94 and 112 and the thickness is between 0.72 and $0.89 \mathrm{~mm}$ due to the variation in density of polyester, cotton and viscose fibres and its proportion in each sample.

\section{Influence of process variables on air permeability of nonwoven fabrics}

The results of air permeability were given in Table 2 and it varies from 60.4 to $94.3 \mathrm{~cm}^{3} / \mathrm{cm}^{2} / \mathrm{s}$. The increase in proportion of the cotton and viscose reduces the air permeability of the fabrics. The equal proportion of fibre increases the air permeability of the multilayer nonwoven fabric (MLNF) due to the distribution of pores from top to bottom layer by decreasing order. This is due to easy bending of cotton and viscose fibres and its surface roughness. Higher proportion of polyester increases the air permeability due to its bulkiness and smoother surface.

\section{Filtration efficiency}

The filtration efficiency of the as prepared samples has been tested in filtration efficiency tester for the particle size of $0.3,0.5,0.7,1$, 2 and 3 microns. The filtration efficiency of the MLNF has been tested for two groups of particle sizes such as 0.3 to 1 and 2 to 3 and the results are also given in table 2 . The proportion of $\mathrm{P} / \mathrm{C} / \mathrm{V}$ as $30 / 40 / 30$ and 40/30/30 gives higher filtration efficiency as $91 \%$ and $93 \%$ respectively with the air permeability of 91.3 and $94.7 \mathrm{~cm}^{3} / \mathrm{cm}^{2} / \mathrm{s}$.

For filtering the particles range from $0.3-1$, the proportion of $\mathrm{P} / \mathrm{C} / \mathrm{V}$ as 20/20/60 gives higher filtration efficiency than other samples and it was shown in Figures 4 and 5. This is due to the serrated cross section of viscose rayon and its close packing density with the air permeability of $87.6 \mathrm{~cm}^{3} / \mathrm{cm}^{2} / \mathrm{s}$.

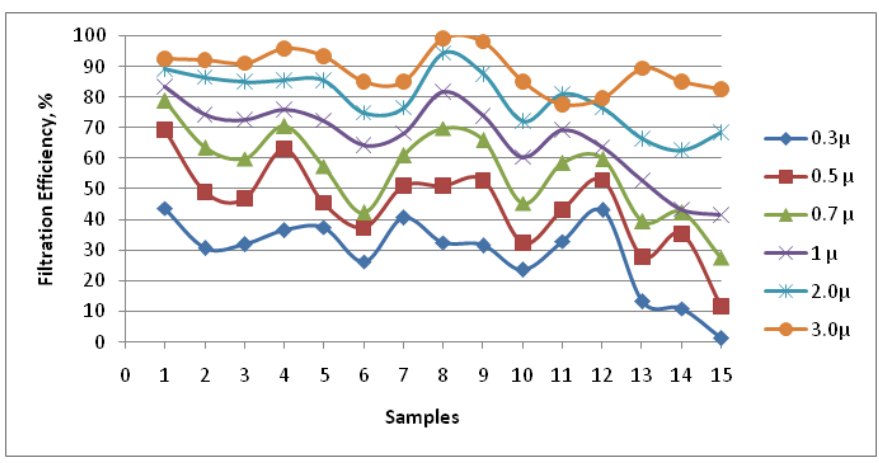

Figure 4 Filtration efficiency of samples with different particles.

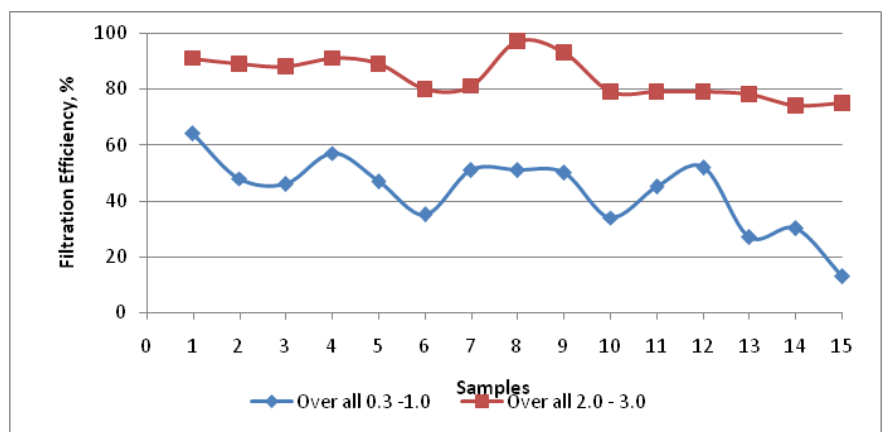

Figure 5 Over all filtration efficiency of samples with different particles (0.3I.0,2-3).

In the principle of depth filtration, the surface properties of the fibres plays major role on filtration and the fibres in each layer of nonwoven fabric and its surface morphology is responsible for filtration performance of MLNF. The serrated cross section of viscose fibre can hold medium to fine particles on its surface and the permeability of the nonwoven fabric will be reduced due blocking of pores, hence the performance of the filter will be reduced if it will be used as a top layer in multi layer nonwoven fabric filter. Polyester fibre on top surface occupies more volume with unit areal density leads to higher air permeability and it helps to ordered filtration of particles from course to fine. The irregular and rough surface of cotton and viscose fibres lead helps to hold more particles at the bottom and it increases filtration efficiency and it was proved in this study. 


\section{Conclusion}

Multilayer nonwoven fabrics were successfully developed and its filtration performance were analysed. The areal density and thickness of the developed nonwoven fabrics were varied based on the proportion of the polyester, cotton and viscose fibres. The proportion of the fibres also affects the air permeability of fabric. The air permeability of the multi layer non woven fabric was reduced when the proportion of cotton and polyester is higher and vice-versa. But the equal proportion of fibres results higher air permeability as $94.7 \mathrm{~cm}^{3} / \mathrm{cm}^{2} / \mathrm{s}$. The minimum air permeability was reported by $20 / 40 / 40$ as $60.4 \mathrm{~cm}^{3} /$ $\mathrm{cm}^{2} / \mathrm{s}$. The variation in proportion of the fibres influences the filtration performance of the nonwoven fabrics. The filtration efficiency for the particles between 0.3 and $1.0 \mu$ is higher for the proportion of $\mathrm{P} / \mathrm{C} / \mathrm{V}$ is $20 / 20 / 60$ with the air permeability of $87.6 \mathrm{~cm}^{3} / \mathrm{cm}^{2} / \mathrm{s}$. Higher proportion of polyester leads to decrease in filtration efficiency. In case of bigger particles such as 1 and $2 \mu$, the $\mathrm{P} / \mathrm{C} / \mathrm{V}$ proportion of $30 / 40 / 30$ and $40 / 30 / 30$ give the filtration efficiency of $97 \%$ and $93 \%$ respectively with highest air permeability values.

\section{Acknowledgments}

None.

\section{Conflicts of interest}

The author(s) declared no potential conflicts of interest with respect to the research, authorship, and/or publication of this article.

\section{References}

1. Tharewal PG, Landage SM, Wasif AI. Application of nonwovens for air filtration. International J Advanced Res. 2013.

2. Das A, Alagirusamy R, Rajan Nagerdra R. Filtration characteristics of spun-laid nonwoven fabrics. Indian J Fibre Tex Res. 2009;34:253-257.

3. Kothari VK, DAS A. Filtration behaviour of woven and nonwoven fabrics. Indian J Fibre Tex Res. 2007;32:214-220.

4. Kothari VK, DAS A, Sarkar A. Effect of processing parameters on properties of layered composite needle punched nonwoven air filters. Indian J Fibre Tex Res. 2007;32:196-201.

5. Woo Sub Shim, Duck Weon Lee. Quality variable of meltblowb submicron filter materials. Indian J Fibre Tex Res. 2013;38:132-137.
6. Hasolli N, Park YO, Rhee YW. Experimental study on filtration performance of flat sheet multiple-layer depth filter media for intake air filtration. Aerosol Science and Technology. 2013;47:1334-1341.

7. Bhatia SK, Smith JL. Geo textile characterisation and pore size distribution: Part I. a review of manufacturing processes. Geosynthetics International. 1996;3(1):85-105.

8. Thangadurai K, Thilagavathi G, Amitava Bhattacharyya. Characterisation of needle-punched nonwoven fabrics for industrial air filter application. The Journal of Textile Institute. 2014;105(12):1319-1326.

9. Wang XY, Gong RH. Thermally bonded nonwoven filters composed of Bi-component polypropylene/polyester fibre.II. Relationships between fabric area density, air permeability and pore size distribution. Journal of applied polymer science. 2006;102(3):2264-2275.

10. Balasubramanian N, Rakshit AK, Desai AN, et al. Avenues for product development in nonwovens. $29^{\text {th }}$ Joint Technological Conference, India.

11. Julien P, Philippe V, Maryline L, et al. Influence of fibre diameter, fibre combinations and solid volume fraction on air filtration properties in nonwovens. Tex Res J. 2012;82(19):1948-1959.

12. Heldmann C. Air filtration composite styructures with superior filtration efficiency and optimised low pressure drop using finest melt blown and staple fibres. Koln, Germany: Proceedings of Filtrex 08; 2008.

13. Rupertseder W, (2008) Improved filter efficiency through integrated nanofibres, In; proceedings of Filtrex08, Koln, Germany.

14. Kunal S, Subhankar M, Mrinal S, et al. Effects of Fibre Diameter Distribution of nonwoven fabrics on its properties. International Journal of Textile Science. 2012;1(1):7-14.

15. Young OP, Hyun SP, Seok JP, et al. Development and Evaluation and mi=ultilayer air filter media. Korean J Chem Engg. 2001;18(6):1020 1024.

16. Lisowski A, Jankowska E, Thorpe A, et al. Performance of textile fibre filter material measured with monodisperse and standard aerosols. Powder Technology. 2001;118(1-2):149-159.

17. Desai AN, Balasubramanian. Critical Factors affecting the properties of thermal bonded nonwovens with special references to cellulosic fibres. Indian J Fibre Tex Res. 1994;19:209-215.

18. Kalaivani D, Arun karthick S, Gobi N. Filtration Properties of staple fibre thermo-bonded nonwoven fabrics. Indian J Fibre Tex Res. 2016;41:6266. 